\title{
INVESTIGATION OF PARAMAGNETIC SATURATION IN LANTHANUM MANGANESE NITRATE
}

\author{
J. FLOKSTRA ${ }^{\ddagger}$, H.C. MEIJER, G.J.C. BOTS and W.A. VERHEIJ \\ Technische Hogeschool Delft, \\ Delft, Nederland \\ and \\ L.C. VAN DER MAREL \\ Technische Hogeschool Twente, \\ Enschede, Nederland
}

Received 10 May 1972

\section{Synopsis}

Paramagnetic saturation of lanthanum manganese nitrate, $\mathrm{La}_{2} \mathrm{Mn}_{3}\left(\mathrm{NO}_{3}\right)_{12} \cdot 24 \mathrm{H}_{2} \mathrm{O}$, has been investigated at liquid $\mathrm{He}$ temperatures in a static as well as a dynamical way. With the aid of the molecular-field theory the Casimir and Du Pré dispersion and absorption curves are adapted explicitly to the phenomenon of saturation. A satisfactory agreement has been observed between theory and experiment.

1. Introduction. The main purpose of the investigations to be described was to verify experimentally some relations that can easily be calculated from classical paramagnetic theory and molecular-field theory. In order to obtain a reasonable degree of saturation in our experiments, lanthanum manganese nitrate (abbreviation $\mathrm{LMnN}$ ) was used, because for the $\mathrm{Mn}^{++}$ion $S=5 / 2$. This ion has the additional advantage that $L=0$, so that no orbital angular momentum has to be taken into account. The influence of paramagnetic saturation on the Casimir and Du Pré formulas was firstly considered by De Haas and Du Pré ${ }^{1}$ ). Their work has been extended by Van den Broek et al. ${ }^{2}$ ). They made use of approximations which could reasonably describe their experiments. In this paper we derive some formulas that describe the experimental results in a more nearly exact way. Two kinds of susceptibility measurements were performed: static measurements by means of a Faraday balance, and dynamic measurements by means of a mutualinductance bridge. In the first set of experiments $\chi(T, H, v=0)$ was determined, in the second set $\chi^{\prime} / \chi_{0}(T, H, v)$ and $\chi^{\prime \prime} \mid \chi_{0}(T, H, v)$ were obtained.

₹ Present address: Twente University of Technology, Enschede, The Netherlands. 
2. Samples. The chemical formula of $\mathrm{LMnN}$ is $\mathrm{La}_{2} \mathrm{Mn}_{3}\left(\mathrm{NO}_{3}\right)_{12} \cdot 24 \mathrm{H}_{2} \mathrm{O}$. It is supposed to have the same structure as cerium magnesium nitrate. The space group of this substance, $R \overline{3}$, was determined by Zalkin $^{3}$ ). There are three $\left[\mathrm{Mn}\left(\mathrm{H}_{2} \mathrm{O}\right)_{6}\right]^{++}$complexes per unit cell. One of the three $\mathrm{Mn}^{++}$ions per unit cell has symmetry $\overline{3}$ (site I), whereas the other two have only symmetry 3 (site II). The molar weight of $\mathrm{LMnN}$ is 1619 , the approximate density $2 \times 10^{3} \mathrm{~kg} / \mathrm{m}^{3}$.

The a.c. measurements were performed perpendicular to the triad axis on a cylinder of $20 \mathrm{~mm}$ length and $6.5 \mathrm{~mm}$ diameter, weighing $1.325 \mathrm{~g}$. For the d.c. measurements a small crystal of $10.1 \mathrm{mg}$ was used, in this case the field pointed along an arbitrary direction.

3. Experimental methods. For the a.c. experiments a Hartshorn mutualinductance bridge was used, which automatically records $\chi^{\prime} v s . H$ and $\chi^{\prime \prime} v s . H$ curves at fixed frequency and temperature $\left.{ }^{4}\right)$. As soon as sufficient graphs of this kind are available, sets of $\chi^{\prime}(v)$ and $\chi^{\prime \prime}(v)$ curves can be constructed. External fields, up to $1.75 \times 10^{6} \mathrm{~A} / \mathrm{m}(22 \mathrm{kOe})$, were generated by a superconducting coil. Frequencies between 1.5 and $3000 \mathrm{~Hz}$ were used, and temperatures were varied between 2.06 and $4.24 \mathrm{~K}$.

From the $\chi^{\prime}(\log v)$ and $\chi^{\prime \prime}(\log v)$ curves, the following information can be obtained: firstly, the limiting value of $\chi^{\prime}$ for $v \rightarrow 0$ gives the information about the degree of paramagnetic saturation. Secondly, the relaxation behaviour as a function of field and temperature can be studied. This subject will be discussed elsewhere ${ }^{5}$ ). Thirdly, the limiting values for $v \rightarrow \infty$ of the $\chi^{\prime}(\log v)$ curves at different $H$ give information about the specific heat of the sample.

The d.c. measurements were performed with a Faraday balance, described by Gijsman $^{6}$ ). The magnetization of $\mathrm{LMnN}$ was determined at six temperatures between 1.2 and $4.2 \mathrm{~K}$, and at field strengths between $1.35 \times 10^{5}$ and $14 \times 10^{5} \mathrm{~A} / \mathrm{m}$ (1.7 to $17.6 \mathrm{kOe})$.

4. Theory. The magnetization $M$ is given by the well-known Brillouin formula:

$$
M=N g \beta S B_{S}(x), \quad x=\mu_{0} g \beta S H / k T,
$$

The field $H$, acting on the ions, is given in the molecular-field theory by:

$$
H=H_{\text {ext }}+\gamma M,
$$

where $H_{\text {ext }}$ is the external field. For small argument $x$,

$$
B_{S}(x)=\frac{S+1}{S} \frac{x}{3} .
$$


The differential susceptibility at zero frequency may be written as:

$$
\chi_{0}(H)=\left(\frac{\partial M}{\partial H_{\mathrm{ext}}}\right)_{T}
$$

Differentiation of (1) with respect to $H_{\text {ext }}$ gives:

$$
\chi_{0}(H)=N g \beta S B_{S}^{\prime}(x)\left(\mu_{0} g \beta S / k T\right)\left[1+\gamma \chi_{0}(H)\right]
$$

and, solving for $\chi_{0}(H)$ :

$$
\chi_{0}(H)=\frac{(C / T) D(x)}{1-(\theta / T) D(x)}
$$

Here, the Curie constant $C$ equals $\mu_{0} N g^{2} \beta^{2} S(S+1) / 3 k, \theta=\gamma C$ and $D(x)$ $\equiv B_{S}^{\prime}(x) / B_{S}^{\prime}(0)$. Then,

$$
K \equiv \frac{\chi_{0}(H)}{\chi_{0}(0)}=D(x) \frac{1-(\theta / T)}{1-(\theta / T) D(x)}
$$

Further, we define a quantity $V$ :

$$
V \equiv \frac{D(x)}{1-(\theta / T) D(x)}
$$

The well-known relations of Casimir and Du Pré for dispersion and absorption are:

$$
\begin{aligned}
& \frac{\chi^{\prime}}{\chi_{0}(H)}=1-F+\frac{F}{1+\omega^{2} \tau^{2}} ; \quad F=\frac{C_{H}-C_{M}}{C_{H}}, \\
& \frac{\chi^{\prime \prime}}{\chi_{0}(H)}=\frac{F \omega \tau}{1+\omega^{2} \tau^{2}} .
\end{aligned}
$$

In (9), $C_{H}$ and $C_{M}$ are the specific heat at constant field and magnetization, respectively. It can easily be shown that:

$$
C_{H}-C_{M}=\mu_{0} C\left(H^{2} / T^{2}\right) \frac{D(x)}{1-(\theta / T) D(x)}
$$


Combining (7), (8) and (11) with (9) and (10), and supposing that $C_{M}=b / T^{2}$, one obtains:

$$
\begin{aligned}
\frac{\chi^{\prime}}{\chi_{0}(0)} & =\frac{b K}{b+\mu_{0} C H^{2} V}+\frac{\mu_{0} C H^{2} V K}{b+\mu_{0} C H^{2} V} \frac{1}{1+\omega^{2} \tau^{2}}, \\
\frac{\chi^{\prime \prime}}{\chi_{0}(0)} & =\frac{\mu_{0} C H^{2} V K}{b+\mu_{0} C H^{2} V} \frac{\omega \tau}{1+\omega^{2} \tau^{2}} .
\end{aligned}
$$

From the formulas (12) and (13) the following predictions can be made.

a. The limiting value of the dispersion curve for $\omega \rightarrow 0$ should be $K$.

b. The limiting value $r$ of the dispersion curve for $\omega \rightarrow \infty$ should be

$$
r=\frac{b K}{b+\mu_{0} C H^{2} V},
$$

so that

$$
\frac{1}{V} \frac{K-r}{r}=\frac{\mu_{0} C}{b} H^{2} .
$$

c. The height $h$ of the absorption curve at $\omega=\tau^{-1}$ should be

$$
h=\frac{1}{2} \frac{\mu_{0} C H^{2} V K}{b+\mu_{0} C H^{2} V},
$$

and consequently:

$$
\frac{1}{V} \frac{2 h}{K-2 h}=\frac{\mu_{0} C}{b} H^{2} .
$$

d. From (14) and (15) it follows that:

$$
K=r+2 h .
$$

The relations (14), (15) and (16) will be compared with the experimental results; besides, both (14) and (15) permit the determination of $b$.

5. Results. 5.1. In static fields. We obtained a set of $M(H)$ curves at six different temperatures between 1.2 and $4.2 \mathrm{~K}$. From the initial slopes $\chi(0)$ the Weiss constant $(\theta=-0.46 \mathrm{~K})$ as well as the Curie constant $\left(C=1.69 \times 10^{-4} \mathrm{Km}^{3} / \mathrm{mol}\right)$ were derived, using the Curie-Weiss law $\chi=C /(T-\theta)$. The value of $C$ agrees satisfactorily with the " $g=2$ value" of the Curie constant, $1.65 \times 10^{-4} \mathrm{~K} \mathrm{~m}^{3} / \mathrm{mol}$. 
From (1), (2) and (3) it is easily seen that:

$$
x=x_{0}+\frac{\theta}{T} \frac{B_{S}(x)}{B_{S}^{\prime}(0)}
$$

if $x=\mu_{0} g \beta S H / k T$ and $x_{0}=\mu_{0} g \beta S H_{\text {ext }} / k T$. Note that $B_{S}^{\prime}(0)$ is simply $(S+1) / 3 S$. Now, for every given point with some $H_{\text {ext }}$ and $T, x$ can be determined either by a simple iteration process or graphically, using the above-mentioned value of $\theta$. Since the magnetization has to obey the law $M=N g \beta S B_{S}(x)$, a plot of $M v s . x$ of all the available experimental points should give a Brillouin curve. Fig. 1

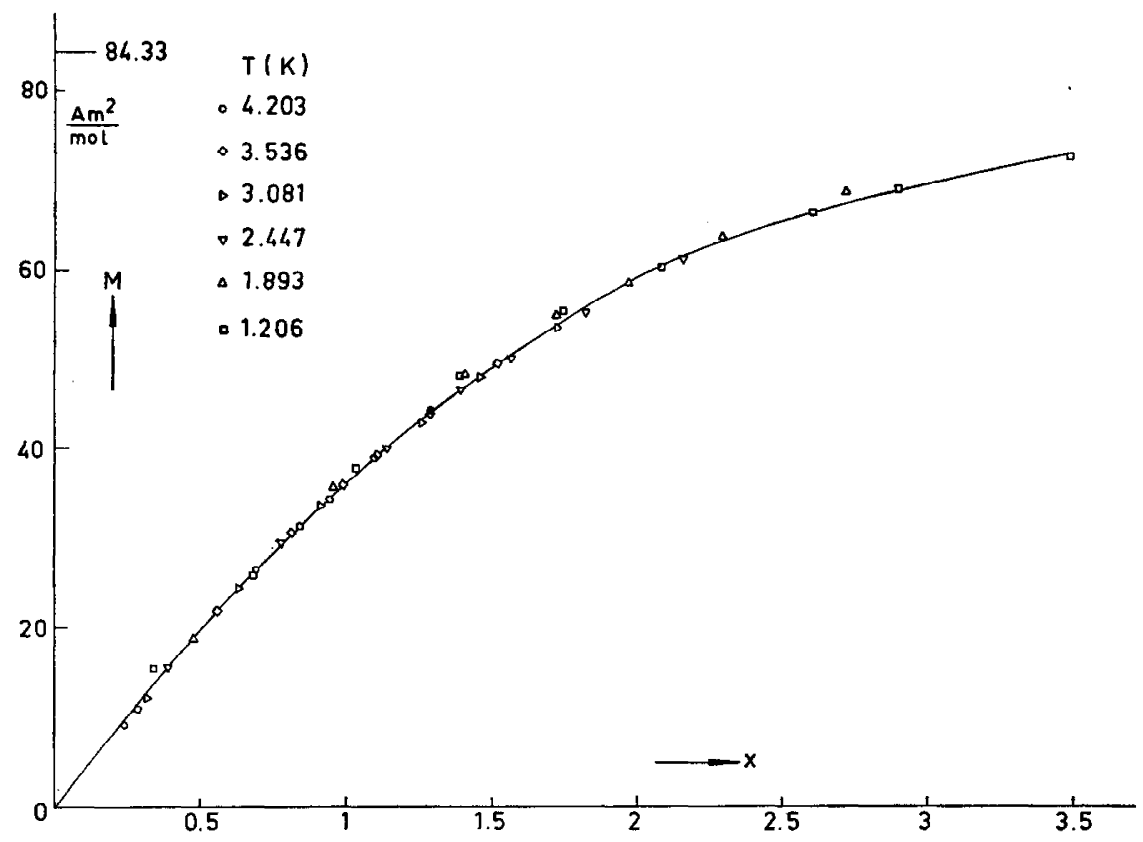

Fig. 1. The static magnetization of lanthanum manganese nitrate as a function of the quantity $x=\mu_{0} g \beta S H / k T$. The experiments obey the law $M=N g \beta S B_{S}(x)$ within the limits of the experimental accuracy.

shows that this is true to a fair degree. The experimental and theoretical values of the constant $\mathrm{Ng} \beta S, 84.3$ and $83.76 \mathrm{Am}^{2} / \mathrm{mol}$, respectively, are equal within the limits of the experimental accuracy. Finally, the relation $\theta=2 z J S(S+1) / 3 k$ gives $z J / k=-0.079 \mathrm{~K}$. In this molecular-field relation, $z$ is the number of nearest neighbours and $J$ the exchange constant. 
5.2. In alternating fields. With this method, a value $\theta=-0.45 \mathrm{~K}$ has been determined for the Weiss constant. The results $\theta=-0.46 \mathrm{~K}$ and $-0.45 \mathrm{~K}$, respectively, may be compared with $\theta=-0.42 \mathrm{~K}$, found by $\mathrm{Mess}^{7}$ ) and $\theta=-0.49 \mathrm{~K}$, found by $\left.\mathrm{Sapp}^{8}\right)$. Rather than with relaxation phenomena, we shall be concerned here with the behaviour of the system at very low and at very high

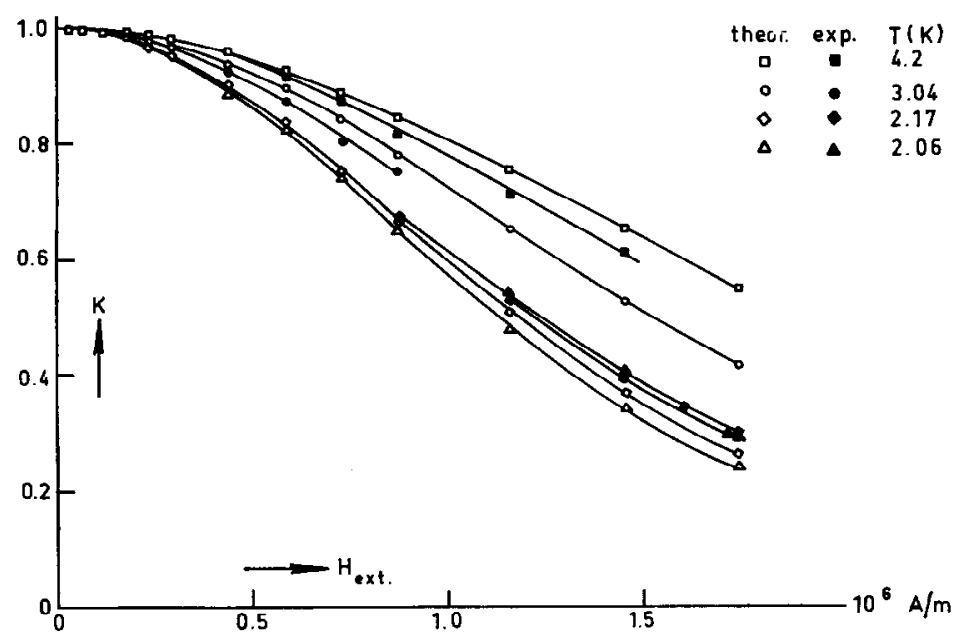

Fig. 2. Experimental and theoretical values of $K=\chi^{\prime}(H) / \chi^{\prime}(0)$ for $\nu \rightarrow 0$ as a function of the magnetic field, at four different temperatures.

frequencies. In fig. 2 the experimental values of $\chi^{\prime}(H) / \chi^{\prime}(0)$ and the theoretical values of $K$ have been plotted as a function of the external field. Agreement between the two kinds of curves is reasonable, considering the difficulty of extrapolating the $\chi^{\prime}(H)$ curve to $\nu=0$.

Fig. 3 shows $\log (K-r) / V r$ and $\log 2 h / V(K-2 h)$ as a function of the logarithm of the applied magnetic field. According to (14) and (15), the slopes of the curves should be 2 . The experimental values are 2.00 and 1.94, respectively. From both graphs the value of $\mu_{0} C / b$ and thus of $b / R$ per gram ion can be determined. For the latter quantity we found $0.081 \mathrm{~K}^{2}$ and $0.085 \mathrm{~K}^{2}$, respectively, giving an average value for $b / R$ of $0.083 \mathrm{~K}^{2}$. Mess ${ }^{7}$ ) quotes $b / R=0.060 \mathrm{~K}^{2}$, and Sapp ${ }^{8}$ ) $0.070 \mathrm{~K}^{2}$.

In fig. 4 a verification is made of (16) by plotting $2 h v s . K-r$. Agreement between experiments and the theoretical (dashed) line is very good, except for the highest field strengths at temperatures above the lambda point $T_{\lambda}(2.17 \mathrm{~K})$. The same remark applies for figs. 2 and 3 . The deviations can be partly understood: firstly, the quantity $r$ is very small and therefore relatively uncertain for large fields. Secondly, since the heat conductivity in the crystal and between liquid and crystal 
is finite, the absorption curve is often broadened, resulting in a relatively small value for $h$. In fact, our measurements do show that the absorption curve becomes progressively broader than the Casimir and $\mathrm{Du}$ Pré formulas predict as the field strength increases. The fact that agreement with theory for $T<T_{\lambda}$ is more satisfactory than for $T>T_{\lambda}$ gives further support to this explanation.

Using the formulas $b=\mu_{0} C h^{2}$ and $\mu h=\alpha k T_{\mathrm{c}}$, where $h$ is the so-called internal field, $\mu$ the magnetic moment per ion, $T_{\mathrm{c}}$ the temperature of the magnetic phase

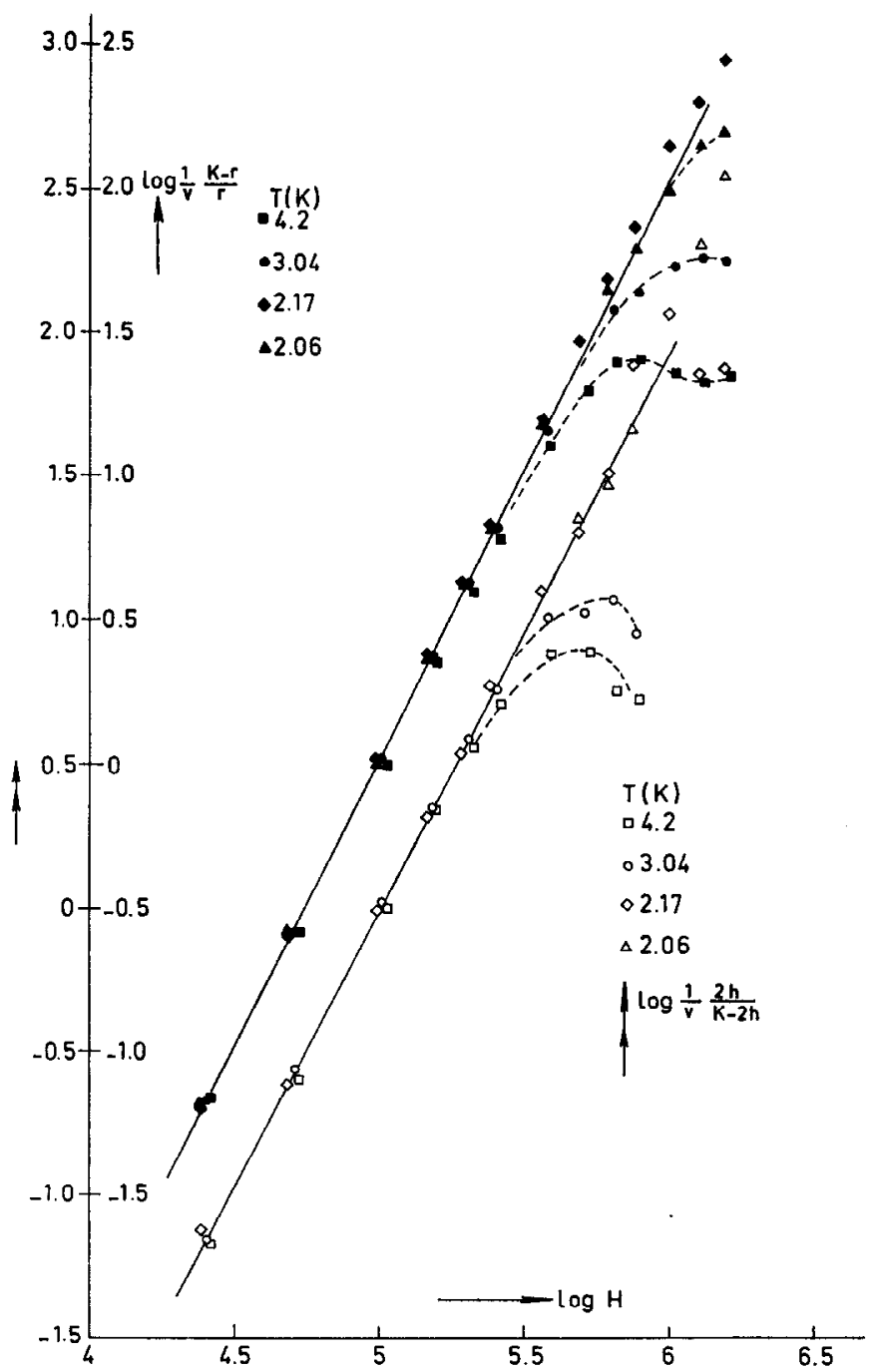

Fig. 3. Plots of $\log (K-r) / V r v s . \log H$ and $2 h / V(K-2 h) v s$. $\log H$, to verify the formulas (14) and (15). The left-hand scale corresponds to the right-hand graph, as indicated by the double arrows. 


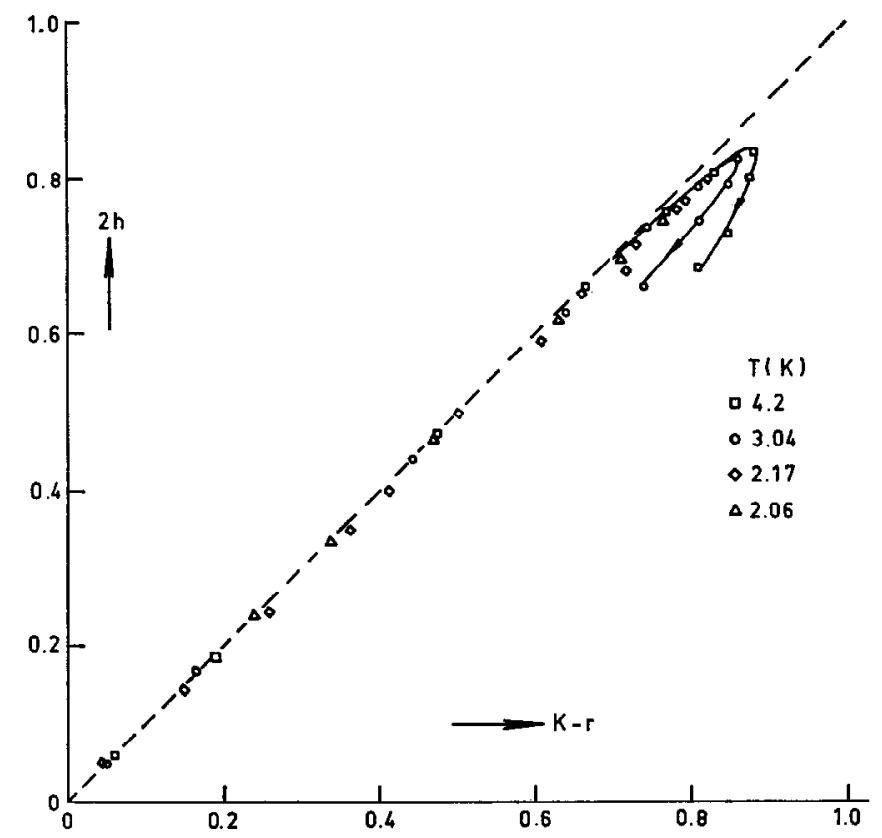

Fig. 4. A verification of formula (16). The experiments should be described by the dashed line $2 h=K-r$.

transition, and $\alpha$ a constant of order unity, one can predict that for two different substances:

$$
b_{1} / b_{2}=T_{\mathrm{c}_{1}}^{2} / T_{\mathrm{e}_{2}}^{2} .
$$

Taking 1 as $\mathrm{LMnN}$ and, for instance, 2 as $\mathrm{CMN}$ (cerium magnesium nitrate), and using the values of $\left.\mathrm{Mess}^{7}\right)$ for $b_{2}, T_{\mathrm{c}_{1}}(0.230 \mathrm{~K})$ and $T_{\mathrm{c}_{2}}(0.0019 \mathrm{~K})$, the left-hand part equals $14 \times 10^{3}$, the right-hand part $15 \times 10^{3}$. This is a good result for such a rough estimation.

One can further estimate the effective number of nearest neighbours $z$ and the average exchange constant $J$, by applying several corrections to the overall value of $\left.b / R^{7}\right)$ to retain the exchange part of the specific heat, and combining the Van Vleck $\left.{ }^{9}\right)$ formula $C_{M} / R=(2 z / 3)[J S(S+1) / k T]^{2}$ for the exchange specific heat with the corrected value of $b / R$ and the relation $z J / k=-0.079 \mathrm{~K}$. The result is: $z=5.0$ and $J=-0.016 \mathrm{~K}$. It should be emphasized that there are two sites for the $\mathrm{Mn}^{++}$ions, so the numbers for $z$ and $J$, mentioned above, are mean values for the two sites.

In conclusion, one may state that the magnetic behaviour of $\mathrm{LMnN}$ can be well described with the molecular-field theory. The deviations between theory and experiment can be understood qualitatively. 
Acknowledgements. We are much indebted to Drs. L.J.M. van de Klundert for his permission to use the improved Hartshorn bridge, and for his guidance during the measurements. The authors are very grateful to Professor B.S. Blaisse for his encouragement and stimulating discussions. Professor J. van den Handel of Leiden University was so kind to permit the measurements with the Faraday balance, and we are grateful to Drs. S.R. Brouwer for his participation in these experiments. Finally, we wish to express our sincere thanks to the technicians of the three laboratories that were involved for their indispensable aid.

\section{REFERENCES}

1) De Haas, W.J, and Du Pré, F.K., Physica 6 (1939) 705 (Kamerlingh Onnes Lab. comm. no. 258a).

2) Van den Broek, J., Van der Marel, L.C. and Gorter, C.J., Physica 27 (1961) 661.

3) Zalkin, A., Forrester, J.D. and Templeton, D.H., J. chem. Phys. 39 (1963) 2881.

4) Van de Klundert, L.J.M. and Van der Marel, L.C. (to be published).

5) Flokstra, J., Verheij, W.A., Van der Marel, L.C., Bots, G.J.C. and Van de Klundert, L.J.M., Phys. Letters 40 (1972) 363.

6) Gijsman, H.M., thesis, Leiden 1958 (unpublished).

7) Mess, K.W., Lagendijk, E., Zimmerman, N.J., Van Duyneveldt, A.J., Giesen, J.J. and Huiskamp, W.J., Physica 43 (1969) 165.

8) Sapp, R.C. and Nelson, D. A., Bull. Amer. Phys. Soc. 11 (1966) 911.

9) Van Vleck, J.H., Phys. Rev. 57 (1940) 426, 1062. 